\title{
Histone H3 Lysine 27
}

National Cancer Institute

\section{Source}

National Cancer Institute. Histone H3 Lysine 27. NCI Thesaurus. Code C116018.

The lysine residue found at amino acid position 27 in the histone H3 protein. Methylation of this residue may be a marker for transcriptionally repressed genes. 